\title{
Recuperación Parcial del Concentrado de la Porquinaza, una Alternativa Ambiental y Económica.
}

\section{Partial Recovery of the Concentrate Food of Manure, an Environmental and Economical Alternative.}

\author{
Eduardo Valencia G. ${ }^{1}$, William Artunduaga $\mathrm{M}^{2}$. y Luz A. Gordillo P. ${ }^{3}$
}

\section{Resumen}

Con el fin de recuperar parte del concentrado presente en las aguas residuales producto del manejo de 400 cerdos de la finca Canaguaros (Rivera Huila), y ser utilizado como alimento para bovinos, se realizaron en laboratorios de la Facultad de Ingeniería una prueba para la separación de la fracción sólida (concentrado) de la líquida mediante un proceso de sedimentación y una prueba de secado del concentrado recuperado por acción del sol; para caracterizar el efluente se tomaron muestras que en el Laboratorio de Aguas de la Universidad Surcolombiana por métodos estandarizados se les determinaron $\mathrm{pH}, \mathrm{DBO}, \mathrm{SS}, \mathrm{N}, \mathrm{P}$, Grasas y Aceites. Los resultados de la caracterización fueron $\mathrm{pH}=$ $6.7, \mathrm{DBO}=3250 \mathrm{mg} / \mathrm{l}, \mathrm{SS}=8750 \mathrm{mg} / \mathrm{l}, \mathrm{G}$ y A $=28.2 \mathrm{mg} / \mathrm{l}, \mathrm{N}=258 \mathrm{mg} / \mathrm{l}$ y $\mathrm{P}=1020 \mathrm{mg} / \mathrm{l}$. El tiempo de sedimentación de 2 horas 30 minutos obtenido, es el parámetro que se recomienda para dimensionar tanques sedimentadores de concentrado. El tiempo de secado del concentrado en un secador parabólico tipo invernadero con piso en concreto es de 8 horas. Se espera recuperar $402 \mathrm{~g} / \mathrm{Kg}$ de concentrado utilizado por cerdo y obtener porcentajes de remoción $\mathrm{DBO}=13 \%, \mathrm{SS}=78 \%, \mathrm{G}$ y A $=$ $75 \%, \mathrm{~N}=42 \%$ y $\mathrm{P}=57 \%$ considerados como buenos.

Palabras Clave: Aguas residuales, porquinaza, concentrado.

\begin{abstract}
In order to recover some of the concentrate food that is present in the wastewater produced by the management of 400 pigs in the farm Canaguaros (Rivera-Huila), and in order to use it as feed for cattle, a test was performed to obtain the separation of the solid fraction (the purine) from the liquid at the laboratories of Universidad Surcolombiana College of Engineering. The test was done through the process of sedimentation and then drying the recovered purine. Some standardized tests were performed at the Laboratory for the Management of Waters to characterize the effluent, and at the same time, it was measured $\mathrm{pH}, \mathrm{BOD}, \mathrm{SS}, \mathrm{N}, \mathrm{P}$, fats and oils. The results of this characterization were: $\mathrm{pH}=$ $6.7, \mathrm{DBO}=3250 \mathrm{mg} / \mathrm{l}, \mathrm{SS}=8750 \mathrm{mg} / \mathrm{l}, \mathrm{G}$ and $\mathrm{A}=28.2 \mathrm{mg} / \mathrm{l}, \mathrm{N}=258 \mathrm{mg} / \mathrm{l}$ and $\mathrm{P}=1020 \mathrm{mg} / \mathrm{l}$. The sedimentation time of 2 hours and 30 minutes achieved was the parameter recommended to size tanks for purine sediments. The time to dry the purine into a parabolic dryer sort greenhouse with concrete floor was 8 hours. On the other hand, into the tanks for sediments, it is expected to recover $402 \mathrm{~g} / \mathrm{kg}$ of the concentrate food per pig, and to obtain removal fraction of $\mathrm{DBO}=13 \%, \mathrm{SS}=78 \%, \mathrm{G}$ and $\mathrm{A}=75 \%, \mathrm{~N}=$ $42 \%$ and $\mathrm{P}=57 \%$ as well.
\end{abstract}

Keywords: Wastewater, manure, concentrates.

1 Magíster en Ingeniería Sanitaria y Ambiental. Docente Universidad Surcolombiana Neiva. Avenida Pastrana Carrera $1^{a}$.eduvale@usco.edu.co 2 Ingeniera Agrícola. Cooperativa Creer en lo Nuestro. Calle $26 N^{o} 10 w-145$. Neiva. . Pollo2541@ hotmail.com

3 Ingeniero Agrícola. Cooperativa Creer en lo Nuestro. Calle $26 N^{o} 10 w-145$. Neiva. Adria207@hotmail.com 


\section{Introducción}

El problema de la disposición sanitaria de los desechos de las explotaciones porcinas es de gran magnitud, por la cantidad de contaminantes que contienen. Pero, es necesario tener en cuenta que aproximadamente dos tercios de los alimentos suministrados a los cerdos se convierten en desechos, de los cuales el $60 \%$ es concentrado que se puede recuperar. Para ello se deben separar los residuos sólidos (concentrado) de la porquinaza, y luego mediante deshidratación por lo general al sol, obtener un producto seco que pueda almacenarse e incorporarse fácilmente a la dieta de otros animales.

La mezcla de residuos sólidos y líquidos que son acarreados por el agua de lavado se conocen como aguas residuales; sus principales ingredientes son las excretas (heces y orina), agua, alimento desperdiciado, cama, suelo y otras partículas. En México para el cálculo de las cantidad de excretas producida se utiliza $6.71 \%$ del peso vivo total de la Granja. (Pérez, 1998).

El problema de la contaminación causada por las excretas porcinas puede convertirse en una oportunidad, en la medida en que se valoren en su justa dimensión como fuente contenedora de energía, materia orgánica y nutriente. Seguir arrojándolas sin ningún control, implica no solo un deterioro del ambiente sino una pérdida de energía y nutrientes lo cual significa pérdida de dinero (Artunduaga y Gordillo, 2009).

Las excretas porcinas, son un subproducto de la producción ganadera, que generalmente se cataloga como residuo. Sin embargo, desde el punto de vista de producción limpia, es un material con numerosos usos, entre ellos; para los residuos sólidos: compost, lombricultura, porquinaza seca (abono), porquinaza en alimentación animal, fertilización sólida; en cuanto a las excretas líquidas: fertilizantes líquidos, producción de energía y biomasa, pastos productivos complementarios (Arias, 1998).

La contaminación producida por una explotación porcina puede variar de acuerdo al estado fisiológico de los animales y al tipo de alimentación utilizada. El grado en que esta contaminación afecta a las fuentes de agua, depende de la cantidad de agua usada, de si se hace separación de sólidos o no y del manejo dado a los residuos. La contaminación promedio producida por día en una explotación porcina para $100 \mathrm{~kg}$ de peso vivo, es $0.25 \mathrm{~kg}$ de DBO, 0,75 kg de DQO, $0.60 \mathrm{~kg}$ de SST, $0.75 \mathrm{~kg}$ de ST y $0.045 \mathrm{~kg}$ de N. (Chara, 1998). La tabla 1, presenta una caracterización de las aguas residuales provenientes de la actividad porcina.

Tabla 1. Características fisicoquímicas de aguas residuales porcinas

\begin{tabular}{|c|c|c|}
\hline Parámetro & Unidad & Valor \\
\hline $\mathrm{pH}$ & unidades & $6-8$ \\
\hline $\mathrm{DBO}$ & $\mathrm{mg} / \mathrm{L}$ & $8000-50000$ \\
\hline $\mathrm{DQO}$ & $\mathrm{mg} / \mathrm{L}$ & $10000-200000$ \\
\hline $\mathrm{ST}$ & $\mathrm{mg} / \mathrm{L}$ & $1200-5000$ \\
\hline $\mathrm{SV}$ & $\mathrm{mg} / \mathrm{L}$ & $500-5000$ \\
\hline $\mathrm{N}$ total & $\mathrm{mg} / \mathrm{L}$ & $1500-5000$ \\
\hline $\mathrm{P}$ & $\mathrm{mg} / \mathrm{L}$ & $1000-3000$ \\
\hline $\mathrm{K}$ & $\mathrm{mg} / \mathrm{L}$ & $1000-3000$ \\
\hline Densidad estiércol & $\mathrm{g} / \mathrm{cm}^{3}$ & $1.01-1.03$ \\
\hline
\end{tabular}

Fuente: Arias, 1998.

Los cerdos consumen alimentos de un alto valor proteico, sin embargo, son ineficientes transformadores y desperdician un alto porcentaje de las proteínas y micronutrientes disponibles en los granos y oleaginosas que forman parte importante de las dietas convencionales en la porcicultura moderna. (Violeta y Dimna, 2001).

La excreta porcina presentan entre un 5 y $30 \%$ de la energía requerida por el animal en la dieta, y esta fracción nutritiva tiene una alta digestibilidad. Dada su condición de monogástrico, el cerdo concentra en las excretas altos niveles de pared celular (44.6\%), lignocelulosa $(24,3 \%)$, lignina $(4,9 \%)$ y hemicelulosa $(20,3 \%)$. También contiene 
otros elementos como plomo, cadmio y arsénico, vitamina A y del complejo vitamínico B (Henning y Flachowsky, 1982). (Ramírez, 2005).

Como una alternativa al manejo de las excretas porcinas en Antioquia se ha utilizado el sistema Cerdos Pastos - Leche. El sistema ha tenido una rápida difusión debido a los múltiples efectos benéficos del estiércol como fertilizante entre los cuales se destacan: su rápida biodegradabilidad, aporte de varios nutrimentos, mejora de las condiciones físicas de los suelos, conservación de la humedad y un incremento de la actividad de la población microbiana del suelo. Reduciendo así los costos de la producción lechera, principalmente por la disminución de la fertilización química y el incremento en la producción de forraje (Rua y Salas, 1998).

La práctica de alimentar rumiantes con las excretas porcinas está ampliamente difundida en países de América Latina. Las excretas se separan por medios mecánicos o manuales, se mezclan con granos y otros ingredientes como las melazas, y llegan a sustituir al grano hasta en un 40\% en las etapas de engorde. (Pérez, 1998). A la fracción sólida de la porquinaza después del secado se le puede adicionar 5\% de melaza para suministrar al ganado de engorde; siendo posible reemplazar el $20 \%$ de la dieta total. El ganado consume bien esa mezcla, sola o combinada con otros productos (Castrillon et. al., 2004).

Sin embargo, el uso de excretas frescas no es totalmente aceptado debido a la supuesta presencia de microorganismos, posiblemente patógenos, tanto para los animales como para el hombre. Para evitar este problema, las excretas y el guano (sólido prensado), ha sido probado en forma de ensilaje solo o combinado con otros ingredientes, seco y húmedo en dietas para ovinos y bovinos; buscando sustituir granos o fuentes proteicas dado su contenido de alrededor del $20 \%$ de proteína cruda. El ensilaje de excretas o guano es un proceso que disminuye las pérdidas de nutrientes, elimina los patógenos, mejora la patabilidad e incrementa el consumo voluntario. Es posible además incorporar otros subproductos agroindustriales como la paja de cereales y la melaza. Sin embargo el ensilaje, da origen a un producto voluminoso relativamente difícil de manipular y con menor contenido de energía. (Herrera y Peralta, 1997).

Como fuente de alimento para rumiantes, es ideal, debido a que su composición química se caracteriza por tener un alto contenido de proteína cruda, fibra cruda, cenizas, y valores bajos de extractos etéreos. Las excretas de cerdo en engorda contienen nitrógeno en forma de proteína cruda $(23.5 \%)$ y en forma de nitrógeno proteico $(66.3 \%)$ (Ramírez, 2005).

Los problemas de riesgo para la salud parecen ser de menor importancia cuando el procesamiento elimina muchos de los riesgos potenciales, por el contrario el procesamiento puede ser benéfico al mejorar la potabilidad, lograr la destrucción de patógenos y el control del olor. Algunas evidencias sugieren que las bacterias patógenas desaparecen a lo largo del tracto digestivo de los rumiantes alimentados con el estiércol seco del cerdo (Castrillon y otros, 2004).

Es posible utilizar diferentes métodos para la separación de la fracción sólida de la liquida de las aguas residuales provenientes de la explotación porcícola, como pantallas estacionarias o cribas ó por separadores de tornillo de prensa. Los primeros, remueven solo parte del agua libre por gravedad y nada de la depositada por capilaridad en las mezclas de sólidos y líquidos. En el segundo caso, se exprime toda el agua libre, más una parte de la depositada por capilaridad, produciendo sólidos secos que se pueden transportar fácilmente y usarse en alimentos balanceados. Estos dos sistemas tienen como desventaja la elevada perdida de nutrimentos cuando los líquidos no son utilizados, la presencia de microorganismos patógenos, elevada inversión inicial así como un alto costo por mantenimiento del mecanismo de separación, además es un equipo recomendado para granjas con grandes instalaciones.(Ramírez, 2005). También se puede realizar esta separación mediante cámaras, las cuales por acción de la gravedad (decantación de sólidos), separan la parte sólida recuperable de las aguas residuales. (Pérez, 1998).

Estos sistemas de secado, pueden ser complementados con secado al sol, se obtiene un producto seco que puede almacenarse e incorporarse fácilmente en una dieta completa, la contaminación del aire es baja y el manejo que se requiere es mínimo. (Ramírez, 2005). La deshidratación se puede producir por cualquiera de las siguientes técnicas: Secado solar en plataforma, Secado solar en eras, Secado solar en invernadero, Secado con equipo y por combustión de algún energético. El tipo invernadero, puede ser una caseta cubierta con plástico en cuyo piso se aloja el estiércol; el piso debe tener un sistema de drenaje para el excedente de agua. La caseta cuenta con un sistema de ventilación natural para desalojar la humedad procedente de la evaporación. Las ventajas de este sistema, son eliminación de bacterias no esporuladas, muerte de huevos y larvas de parásitos e insectos, detención de los procesos de oxidación o fermentación, estabilización del contenido nutricional, disminución del peso y volumen del estiércol, eliminación de malos olores (como producto final). Las principales desventajas son: aumento de la pérdida de N por volatilización 
(hasta un 60\%), proliferación de moscas y otros insectos, generación de olores desagradables durante el proceso, necesidad de un terreno amplio. (Salazar, 2005).

El Secado artificial, es un método de separación y de control de la carga bacteriana, debido a que las altas temperaturas que se alcanzan con el tratamiento, eliminan patógenos y las heces secas son inodoras. Este procedimiento requiere el uso de equipo caro y los costos de energía, recolección y transporte de las excretas hacia los deshidratadores son elevados. (Ramírez, 2005).

Como parte del concentrado contenido en la porquinaza puede ser recuperado, en este estudio se realizaron pruebas de sedimentación con el fin de determinar el tiempo requerido para diseñar tanques sedimentadores y pruebas de secado para dimensionar secadores parabólicos. Presentando una alternativa ambiental y económica para la problemática de contaminación por las excretas porcinas, al convertirse en una oportunidad, en la medida en que se valoren como fuente de alimentación para otras especies animales.

\section{Metodología}

\subsection{Localización}

El estudio se realizó en la Finca los Canaguaros localizada al Noroccidente del Municipio de Rivera, en jurisdicción de la vereda los Medios, región norte del departamento del Huila, a una altura de 700 m.s.n.m. y temperatura media de $25^{\circ} \mathrm{C}$. Las pruebas y análisis de laboratorio, en el edificio de Ingeniería de la Universidad Surcolombiana de Neiva - Huila., con 430 m.s.n.m. y temperatura media de $28^{\circ} \mathrm{C}$.

2.2 Caracterización de las aguas residuales: Del efluente de la porqueriza se tomó una muestra de 5 litros en un balde calibrado, el día 18 de marzo de 2009, a las 3:30 pm, y las temperaturas ambiente y del agua. De esta agua, se tomaron 4 muestras de 500 mililitros cada una, en recipientes dados por el laboratorio de Calidad de Aguas de la Universidad Surcolombiana. Las muestras fueron entregadas al Laboratorio, a las 4:45 pm del mismo día. En el laboratorio, dos de las muestras se colocaron a sedimentar durante 2 horas (muestra de agua decantada). A las 4 muestras ( 2 muestras de agua agitada y 2 muestras de agua decantada) se les determinaron los siguientes parámetros: $\mathrm{pH}, \mathrm{DBO}, \mathrm{SS}, \mathrm{G}$ y A (grasas y aceites), $\mathrm{N}$ (Nitrógeno amoniacal) y $\mathrm{P}$ (fosfatos). Para las temperaturas ambiente y del agua residual se utilizó un termómetro, para el pH un peachímetro o potenciómetro, para determinar la DBO se utilizó el método titulométrico Winkler, para los SS el método gravimétrico, para las G y A los métodos gravimétrico y solvente-cloroformo, para $\mathrm{N}$ el titulométrico-ácido bórico, y para el $\mathrm{P}$ el colorimétrico-cloruro estagnoso.

2.3 Pruebas de sedimentación. Las pruebas se realizaron en el Laboratorio de Construcciones de la Facultad de Ingeniería, utilizando un recipiente de vidrio $(e=5 \mathrm{~mm})$ en forma de prisma rectangular, de largo $(\mathrm{L}=35 \mathrm{~cm})$, ancho $(\mathrm{a}=25 \mathrm{~cm})$ y altura $(\mathrm{h}=23 \mathrm{~cm})$. (Ver figura 1$)$. Se tomaron datos cada 15 minutos durante 4 horas y un dato después de 24 horas, de la altura de la parte turbia (ht) del agua, hasta que ht presentó un valor constante, utilizando como método la observación directa del color. (Ver figura 2). Para las pruebas, se agitó el material y se llenó el recipiente hasta una altura ha $=20 \mathrm{~cm}$.

La primera prueba se realizó el día 11 de abril de 2009, con aguas residuales provenientes de la porqueriza de la Finca Canaguaros; y la segunda, el día 8 de mayo de 2009, con una mezcla de $3 \mathrm{Kg}$ de concentrado para cerdos disueltos en 20 litros de agua.

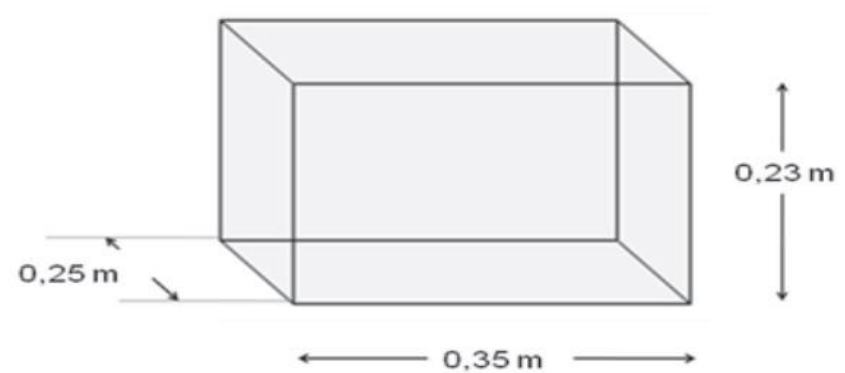

Figura 1. Recipiente ensayo de sedimentación

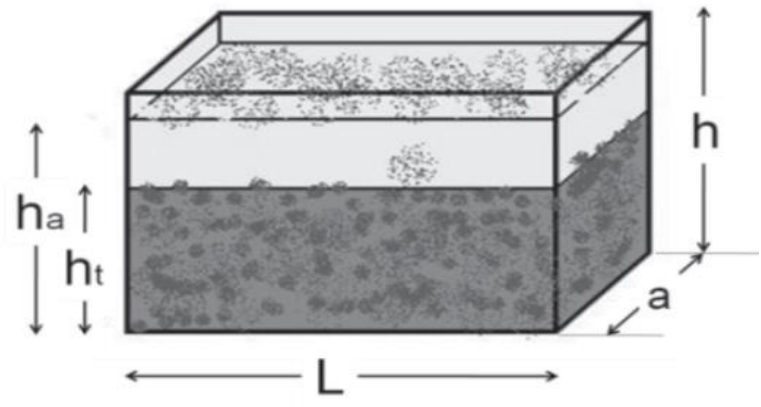

Figura 2 Ensayo de sedimentación 
2.4 Prueba tiempo de secado del concentrado: El objetivo de la prueba era determinar mediante dos tratamientos (Concentrado escurrido y sin escurrir), el tiempo de secado de 2 kilos de concentrado para cerdos diluidos en 2 litros de agua, sobre diferentes superficies y con diferentes contenidos de agua. Las pruebas se realizaron el día 14 de Junio de 2009, en un pasillo del edificio de la Facultad de Ingeniería, con exposición directa de sol. Como Tratamientos: T1 escurrido, a la muestra se le retiró el exceso de agua mediante escurrimiento; T2 sin escurrir, muestra del concentrado diluido en agua. Las pruebas se iniciaron a las 8:00 am, colocando las muestras con un espesor de $3 \mathrm{~cm}$, sobre diferentes superficies (Ver tabla 2). Cada 30 minutos se observaba y palpaba el concentrado, hasta encontrarse seco. La temperatura ambiente promedio durante el día fue de $28^{\circ} \mathrm{C}$.

Tabla 2. Superficies utilizadas para la prueba de secado del concentrado.

\begin{tabular}{|l|c|c|}
\hline \multicolumn{1}{|c|}{ Superficie } & Escurrida & Sin escurrir \\
\hline Piso en concreto & $\mathrm{X}$ & $\mathrm{X}$ \\
\hline $\begin{array}{l}\text { Piso en concreto y secador } \\
\text { parabólico }\end{array}$ & $\mathrm{X}$ & $\mathrm{X}$ \\
\hline Bandeja plástica perforada & & $\mathrm{X}$ \\
\hline Plástico & $\mathrm{X}$ & \\
\hline Recipiente en vidrio & & $\mathrm{X}$ \\
\hline
\end{tabular}

2.5 Eficiencia de remoción de contaminantes: Para el cálculo de la eficiencia de remoción de contaminantes en el sedimentador, se utilizaron los datos obtenidos del laboratorio (agua agitada y agua decantada).

\section{Resultados}

3.1 Caracterización de las aguas residuales: La tabla 3, muestra la caracterización del agua residual proveniente del lavado de la porqueriza, finca Canaguaros.

Tabla 3. Caracterización del agua residual porcina, finca los Canaguaros.

\begin{tabular}{|l|c|c|c|}
\hline \multicolumn{1}{|c|}{ Parámetro } & Unidad & RESULTADO & RESULTADO N $\mathbf{0 0 2 6}^{\circ}$ \\
\hline Temperatura ambiente & ${ }^{\circ} \mathrm{C}$ & 30 & 30 \\
\hline Temperatura muestra & ${ }^{\circ} \mathrm{C}$ & 22 & 6.8 \\
\hline $\mathrm{pH}$ & Unidades & 6.7 & 2820 \\
\hline DBO & $\mathrm{mg} / \mathrm{L}$ & 28.2 & 6.9 \\
\hline Grasas y Aceites & $\mathrm{mg} / \mathrm{L}$ & 8750 & 1960 \\
\hline Sólidos Suspendidos & $\mathrm{mg} / \mathrm{L}$ & 258 & 440 \\
\hline Nitrógeno Amoniacal & $\mathrm{mg} / \mathrm{L} \mathrm{NH}_{3} \mathrm{~N}$ & 1020 & \\
\hline Fosfatos & $\mathrm{mg} / \mathrm{L} \mathrm{PO}_{4}$ & \\
\hline $\begin{array}{l}\text { OBSERVACIONES: } \\
\text { Muestra N } \\
\text { Muestra } \mathrm{N}^{\circ} \text { 0025 corresponde a la muestra de agua agitada. }\end{array}$ \\
\hline
\end{tabular}

Prueba de sedimentación: La prueba de sedimentación $\mathrm{N}^{\circ} 1$ (agua residual proveniente de la porqueriza), dio como resultado, un tiempo de sedimentación de 2 horas y 30 minutos para el concentrado que se puede recuperar y una altura $\mathrm{h}=5 \mathrm{~cm}$ medidos a partir del fondo. (Ver tabla 4 y figuras 3 y 4). La prueba de sedimentación $\mathrm{N}^{\circ} 2$ (Concentrado disuelto en agua), dio como resultado, un tiempo de sedimentación de 1 horas y 30 minutos y una altura $\mathrm{h}=7 \mathrm{~cm}$ medidos a partir del fondo. 
Tabla 4. Prueba de sedimentación con aguas residuales de la porqueriza.

\begin{tabular}{|c|c|c|c|}
\hline TIEMPO (MINUTOS) & $\begin{array}{c}\text { SEDIMENTACIÓN ht } \\
(\mathbf{c m})\end{array}$ & TIEMPO (MINUTOS) & $\begin{array}{c}\text { SEDIMENTACIÓN ht } \\
(\mathbf{c m})\end{array}$ \\
\hline Inicio & 0 & 105 & 10.70 \\
\hline 15 & 20 & 120 & 8.80 \\
\hline 30 & 19.60 & 135 & 7 \\
\hline 45 & 18 & $\mathbf{1 5 0}$ & $\mathbf{5}$ \\
\hline 60 & 16 & 165 & 5.10 \\
\hline 75 & 15.70 & 180 & 5 \\
\hline 90 & 11.80 & 195 & \\
\hline
\end{tabular}

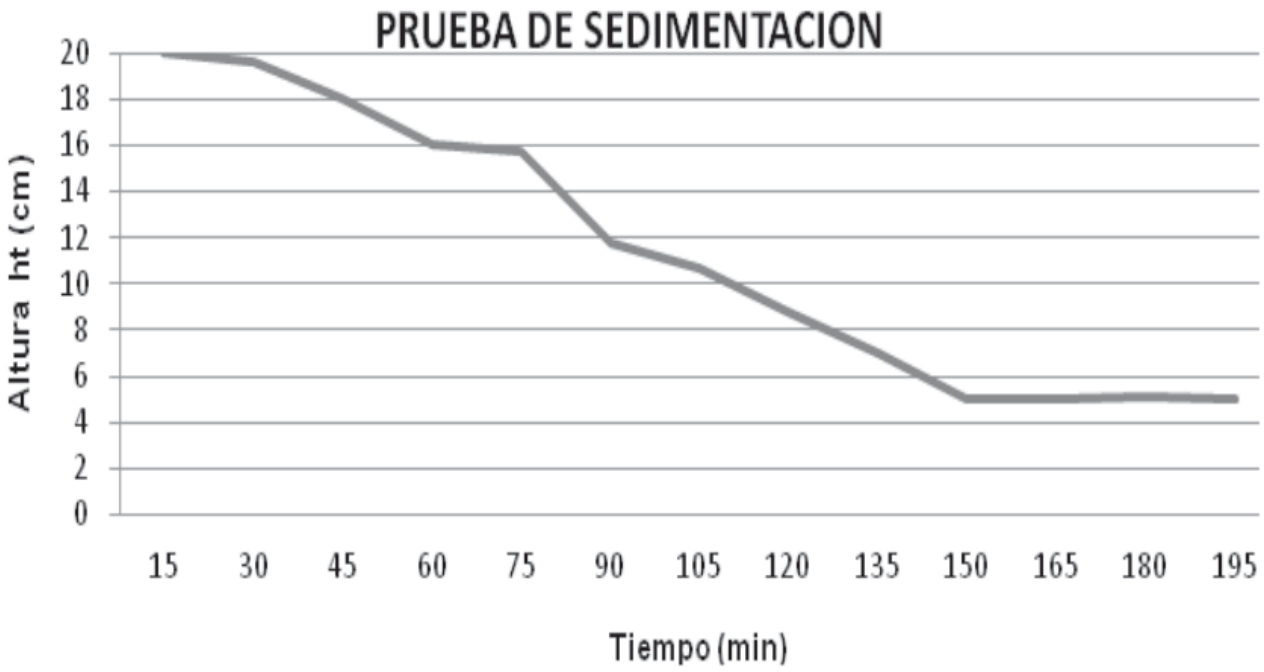

Figura 3. Resultados prueba sedimentación

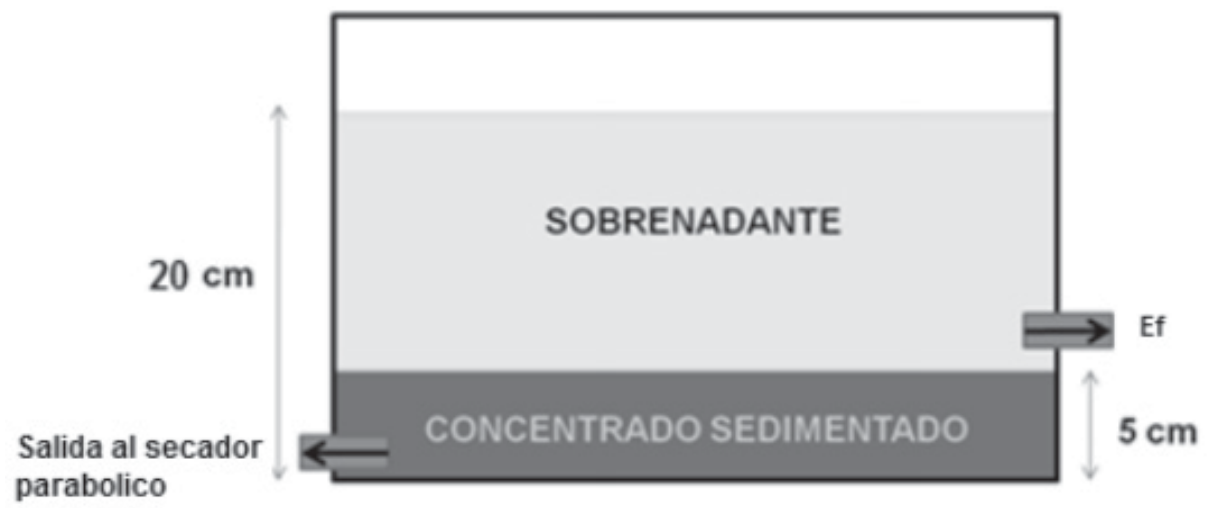

Figura 4. Altura del concentrado sedimentado 
3.3 Prueba tiempo de secado del concentrado: En la tabla 5, se presentan los resultados del tiempo de secado. La superficie con menor tiempo de secado fue el piso en concreto y secador parabólico, con 5 horas 15 minutos en el tratamiento escurrido y 8 horas en el tratamiento sin escurrir.

Tabla 5. Prueba de secado del concentrado recuperado

\begin{tabular}{|l|c|c|}
\hline \multicolumn{1}{|c|}{ Superficie } & Tratamiento & Ts \\
\hline \multirow{2}{*}{ Piso en concreto } & Escurrida & $7.5 \mathrm{~h}$ \\
\cline { 2 - 3 } & Sin escurrir & $9.5 \mathrm{~h}$ \\
\hline $\begin{array}{l}\text { Piso en concreto y secador } \\
\text { parabólico }\end{array}$ & Escurrida & $5 \mathrm{~h} 15 \mathrm{~min}$ \\
\hline Bandeja plástica perforada & Sin escurrir & $8 \mathrm{~h}$ \\
\hline Plástico & Sin escurrir & $12 \mathrm{~h}$ \\
\hline Recipiente en vidrio & Escurrida & $36 \mathrm{~h}$ \\
\hline
\end{tabular}

\subsection{Eficiencias de remoción de contaminantes en el sedimentador}

La tabla 6, presenta los resultados de remoción de contaminantes obtenidos en la prueba $\mathrm{N}^{\circ} 1$, de acuerdo a los resultados de los análisis de laboratorio.

Tabla 6. Eficiencias de remoción de contaminantes en la prueba $\mathrm{N}^{\circ} 1$.

\begin{tabular}{|c|c|c|c|}
\hline Parámetro & A.R. agitada & A.R. sedimentada & \% Remoción \\
\hline DBO (mg/L) & 3250 & 2820 & 13 \\
\hline SS (mg/L) & 8750 & 1960 & 78 \\
\hline G y A (mg/L) & 28.2 & 6.9 & 75 \\
\hline N (mg/L $)$ & 258 & 149 & 42 \\
\hline P $(\mathrm{mg} / \mathrm{L})$ & 1020 & 440 & 57 \\
\hline
\end{tabular}

En el sedimentador, se esperan remociones similares a las logradas en la prueba $\mathrm{N}^{\circ} 1, \mathrm{DBO}=13 \%, \mathrm{SS}=78 \%, \mathrm{G}$ y $\mathrm{A}=75 \%, \mathrm{~N}=42 \%$ y $\mathrm{P}=57 \%$. Según los resultados, un sedimentador es muy eficiente para la remoción de SS, G y A, N y P, y poco eficiente para la DBO.

\section{Conclusiones}

La sedimentación es una buena alternativa para recuperar parte del concentrado contenido en la porquinaza. Los sedimentadores se pueden diseñar con tiempos de retención hidráulica iguales a 2 horas y 30 minutos.

Para disminuir los riesgos microbiológicos del concentrado recuperado, una alternativa es su secado al sol. El secador de mayor eficiencia, fue el secador parabólico con piso en concreto, el tiempo de secado de 8 horas para concretado recuperado sin escurrimiento, a una temperatura ambiente promedio de $28^{\circ} \mathrm{C}$.

Con el uso de sedimentadores, se espera recuperar $402 \mathrm{~g} / \mathrm{Kg}$ del concentrado utilizado para alimentar a un cerdo de $43 \mathrm{~kg}$ de peso.

El uso de sedimentadores para recuperar parte del concentrado contenido en la porquinaza es una buena alternativa económica para las explotaciones porcinas, por cuanto el concentrado recuperado puede ser utilizado para alimentar otras especies animales como los bovinos. Y una muy buena alternativa ambiental, las remociones de $78 \%$ de SS, $75 \%$ de G y A, $42 \%$ de N y $57 \%$ de P, pueden considerarse altas; y $13 \%$ de DBO aceptable para un sedimentador. 


\section{Referencias Bibliográficas}

1. Arias C., A., 1998 Tasas retributivas para el control de la contaminación del agua en Colombia. En Seminario Internacional Contaminación y Reciclaje en la Producción Porcina Aspectos Legales, Técnicos y Económicos. CIPAV, Cali, pp 27 - 34.

2. Artunduaga M,, W., Gordillo P., L. A., 2009. Diseño de un Sistema de Tratamiento para los Residuos Provenientes de la Explotación Porcícola, los Canaguaros Vereda los Medios; Rivera Huila. Trabajo de Grado, Programa de Ingeniería Agrícola, Universidad Surcolombiana. Neiva. 63 pp.

3. Chará J., D., 1998. Potencial de las Excretas Porcinas para Uso Múltiple y los Sistemas de Descontaminación Productiva. En Seminario Internacional Contaminación y Reciclaje en la Producción Porcina Aspectos Legales, Técnicos y Económicos. CIPAV, Cali, pp 49 - 57.

4. Castrillon Q., O., Jiménez P., R. A., Bedoya M, O., 2004. Porquinaza en la Alimentación Animal. Revista Lasallista de Investigación, junio de 2004/ Vol 1, número 001. Corporación Universitaria Lasallista. Medellín. pp $72-76$

5. Herrera C., Ch., Peralta A., M., 1997. Valoración de las excretas porcinas. Consultado el 6 de marzo de 2009. http.//www.porcicultura.com.

6. Pérez E., R., 1998. Porcicultura Intensiva y Medio Ambiente en México Situación Actual y Perspectiva. En Seminario Internacional. Contaminación y Reciclaje en la Producción Porcina Aspectos Legales, Técnicos y Económicos. CIPAV, Cali, pp 1 - 17.

7. Ramírez H., G., 2005. Manejo de Excretas Porcinas, Sistemas Convencionales y Alternativos. Consultado el 7 marzo de 2009. http://www.Porcicultura.Com - artículos-manejo de excretas porcinas, sistemas convencionales y alternativos.

8. Rua G., A., Salas T., S., 1998. Caracterización del Sistema de Manejo de Porquinaza en Varias Explotaciones Porcinas del Norte de Antioquia. En Seminario Internacional Contaminación y Reciclaje en la Producción Porcina Aspectos Legales, Técnicos y Económicos. CIPAV, Cali.,pp 35 - 40

9. Salazar G., G., 2005. Compendio de Tecnologías para el Manejo y Utilización de las Excretas de Granjas Porcícolas. CECEJ-INIFAP. (Parte 2/3).

10.Violeta E., E., Dimna E., A. H., 2001. Tratamiento de Efluentes Porcícolas en Granjas de Traspatio. Consultado el 7 marzo de 2009. http://www.Porcicultura.Com - Artículos - Tratamiento de Efluentes Porcícolas en Granjas de Traspatio. 